\title{
Canal mandibular bífido. Presentación de una serie de casos
}

\section{Bifid mandibular canal. Case series report}

\section{Guzmán Zuluaga CL*, Guzmán Zuluaga IC**, Ardila Medina, CM***}

\section{RESUMEN}

La ubicación y configuración de las variaciones del canal mandibular son relevantes en diferentes procedimientos clínicos de la mandíbula. En esta serie de casos un radiólogo evaluó el recorrido del canal mandibular identificando la presencia y tipos de canales bífidos. Se observaron cuatro pacientes con canal mandibular bífido tipo retromolar y tres sujetos con canal bífido tipo anterior sin convergencia. En otro paciente se encontraron forámenes dentarios bífidos bilaterales. La Tomografía Computarizada de Haz de Cono, permite la identificación oportuna de condiciones preexistentes como variaciones en la morfología natural de los canales mandibulares, previniendo efectiva y precozmente las consecuencias neurológicas que se derivan de una lesión del nervio dentario inferior.

Palabras clave: Canal bífido mandibular, tomografía computarizada de haz de cono.

\section{SUMMARY}

The location and configuration of the mandibular canal variations are relevant in different clinical procedures of the jaw. In this case series, a radiologist assessed the mandibular canal route identifying the presence and types of bifid canals. Four patients with type bifid mandibular retromolar canal and three subjects with type bifida anterior canal without convergence were observed. In one patient, bilateral bifid tooth foramina was found. Computed Tomography Cone Beam allows identification of preexisting conditions such as variations in the natural morphology of the mandibular canals, preventing effective and early neurological consequences arising from alveolar nerve injury.

Key words: Bifid mandibular canal, cone beam.

Fecha de recepción: 25 de marzo de 2011.

Aceptado para publicación: 30 de marzo de 2011.

Guzmán Zuluaga CL, Guzmán Zuluaga IC, Ardila Medina, CM. Canal mandibular bífido. Presentación de una serie de casos $A v$. Odontoestomatol 2012; 28 (2): 71-75.

* Radióloga Oral y Maxilofacial. Profesora Universidad de Chile.

** Periodoncista. Profesora Asistente Universidad de Antioquía.

*** Profesor Asociado, Director Posgrado de Periodoncia. Facultad de Odontología Universidad de Antioquía. Grupo de Epidemiología Universidad de Antioquía. 


\section{INTRODUCCIÓN}

El canal mandibular (CM) se extiende desde el foramen mandibular hasta el foramen mentoniano, conteniendo el paquete vasculonervioso alveolar inferior que inerva e irriga los dientes inferiores. La ubicación y configuración de las variaciones del CM son relevantes en procedimientos clínicos de la mandíbula. La radiografía panorámica, la tomografía computarizada y la tomografía computarizada de haz de cono (TCHC), permiten observar algunas variaciones del CM, aunque por sus características técnicas la panorámica es insuficiente para la detección de variantes anatómicas en los canales mandibulares (1). Por el contrario, la TCHC permite la identificación oportuna de condiciones preexistentes como variaciones en la morfología natural de los CM, previniendo efectiva y precozmente las consecuencias neurológicas que se derivan de una lesión del nervio dentario en procedimientos quirúrgicos como exodoncias y colocación de implantes, explicando además la etiología del fracaso en procedimientos anestésicos (1). Cuando se utilizan radiografías panorámicas, la prevalencia de canal mandibular bífido va desde el 0,08\% (2) hasta el $0,95 \%$ (3). Sin embargo, la frecuencia de esta variación anatómica aumenta al $64.8 \%$ cuando se utiliza TCHC (4).

El objetivo de este artículo es presentar una serie de casos en donde se observan diferentes clases de canales dentarios bífidos.

\section{SERIE DE CASOS}

En este artículo se presentan ocho pacientes que solicitaron examen radiológico con fines diagnóstico. Se efectuaron adquisiciones volumétricas por medio de TCHC, utilizando un equipo Siemens Sirona, modelo Galileos Comfort. Los parámetros del equipo incluyen volumen de radiografías $15 \times 15 \times 15 \mathrm{~cm}$, resolución $3 \mathrm{D}$, longitud de contorno isótropa en vóxeles 0,3-0,15 mm, duración de radiografía/tiempo exposición $14 \mathrm{~s} / 2-6 \mathrm{~s}$, duración de la reconstrucción 2,5-4,5 minutos, kilovoltaje de $85 \mathrm{kV}$ y una dosis efectiva de $29 \mathrm{uSv} / 68 \mathrm{uSv}$ ( $21 \mathrm{~mA}, 85 \mathrm{kV})$. Este equipo está provisto del programa Galaxis para diagnóstico 3D.
Un radiólogo observó el recorrido del CM evaluando la presencia y tipos de canales bífidos. Primero, se utilizó la ventana panorámica, fijando la ventana de corte en la imagen panorámica en el centro de la rama mandibular a la altura de la língula. Luego, se examinó la ventana correspondiente al plano sagital para evaluar la presencia de CM bífido haciendo un barrido desde el borde anterior de la rama mandibular hasta la altura del segundo molar inferior, con proyecciones superficiales y profundas en el mismo plano, modificando la angulación y la posición del corte en sentido lateromedial. Posteriormente, en los cortes coronales, se realizó un análisis en sentido anteroposterior evaluando la presencia de CM bífido tipo vestibular y lingual. Este procedimiento se efectuó en ambos lados de la mandíbula. Para la clasificación de los canales bífidos se utilizaron los parámetros indicados por Naitoh y colaboradores (4). Los resultados fueron registrados en una ficha clínica diseñada para tal efecto.

Cuatro pacientes presentaron CM bífido tipo retromolar; en todos los casos la variante accesoria emergió en la zona distal al tercer molar (Figuras 1- 4). En la figura 3 se aprecia la relación de la rama accesoria del CM con el tercer molar. Adicionalmente, en tres sujetos se observó CM bífido tipo anterior sin convergencia (Figuras 5-7) y en otro paciente se

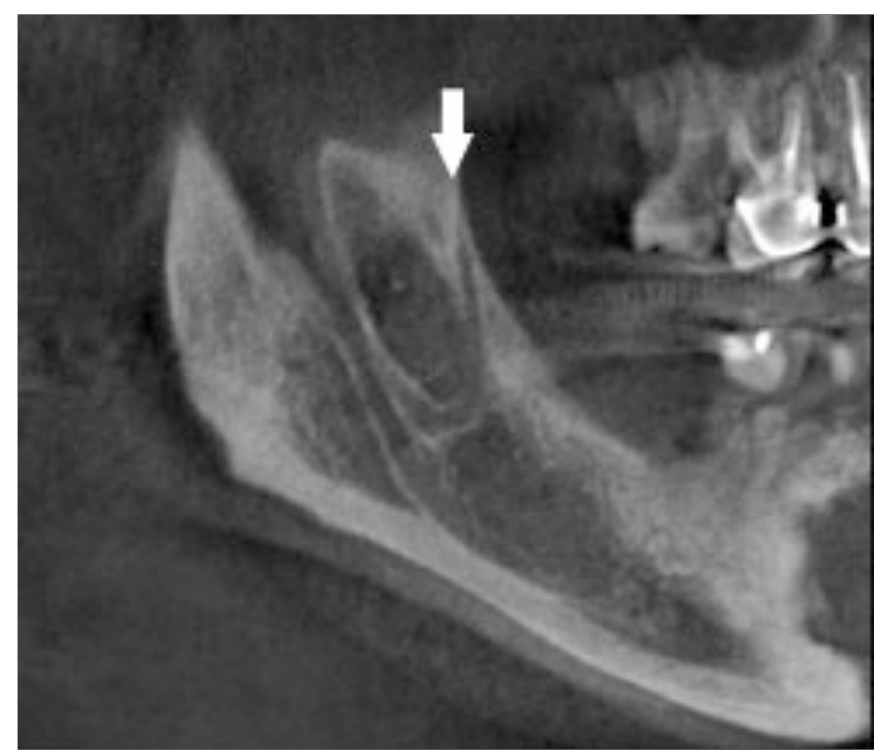

Fig. 1. Bifurcación del Canal Dentario Inferior tipo Retromolar, emergiendo en zona distal al tercer molar. 


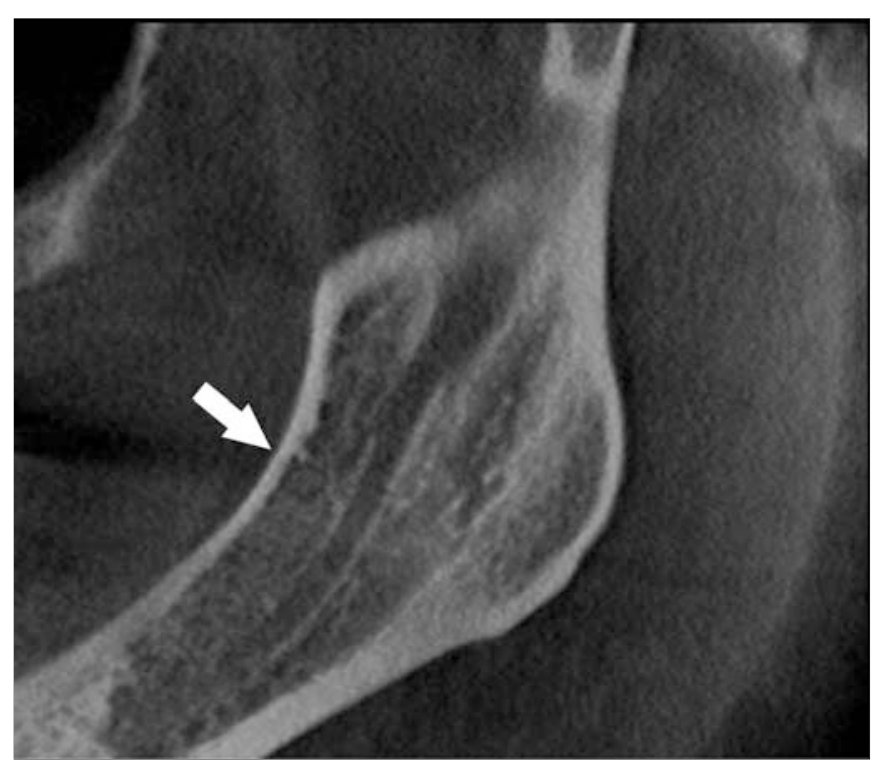

Fig. 2. Canal dentario inferior bífido tipo retromolar izquierdo, con emergencia en zona del tercer molar.

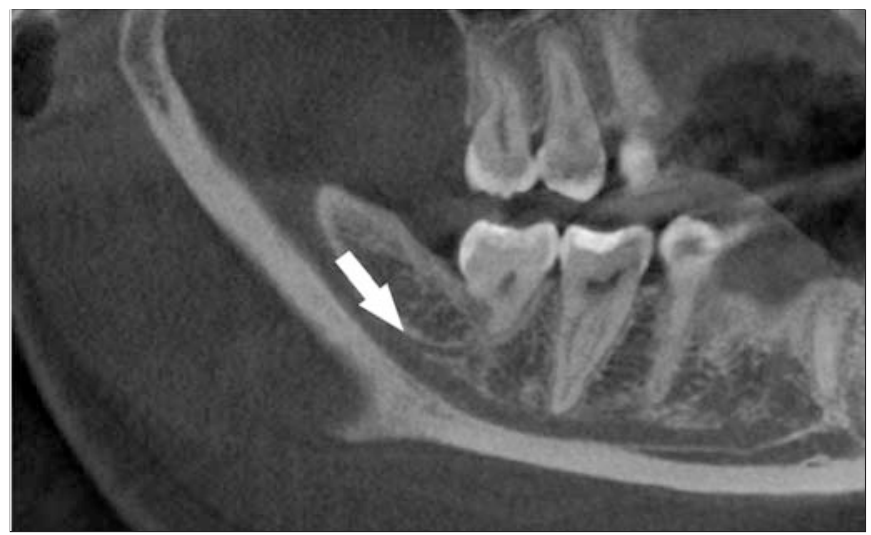

Fig. 3. Canal dentario bífido tipo retromolar. Se aprecia la relación de la rama accesoria del canal con relación al tercer molar permanente.

observaron forámenes dentarios bífidos bilaterales (Figura 8).

\section{DISCUSIÓN}

Los resultados de estudios anatómicos y radiológicos previos indican una variación apreciable del curso del CM. Chávez-Lomeli y cols. (5), indicaron que durante el desarrollo embriológico tres canales separados se fusionan para formar un solo canal. Los

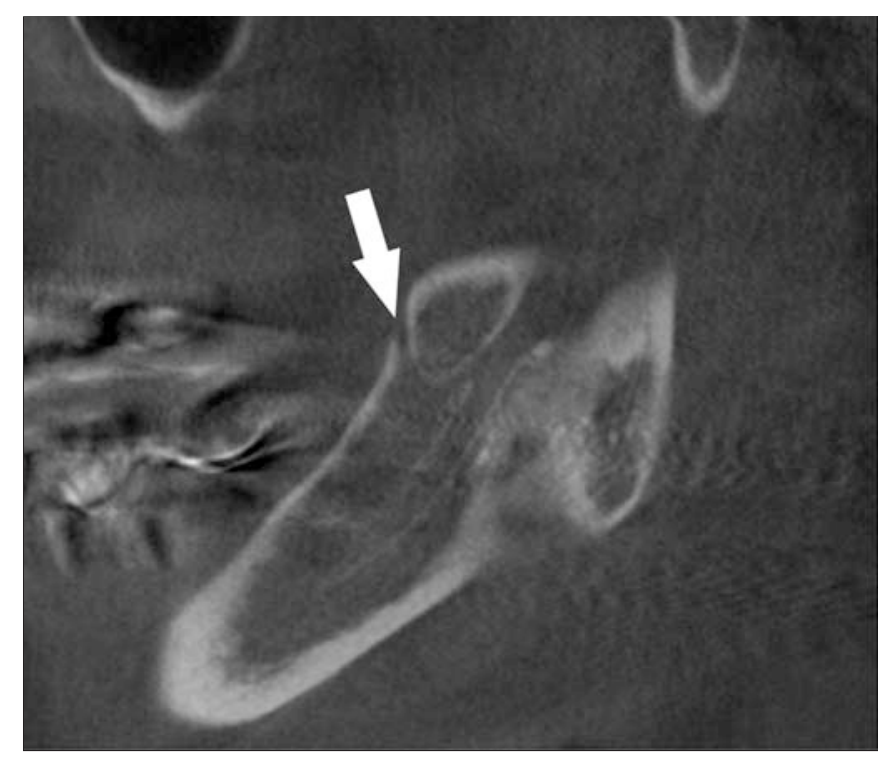

Fig. 4. Canal dentario bífido tipo retromolar izquierdo.

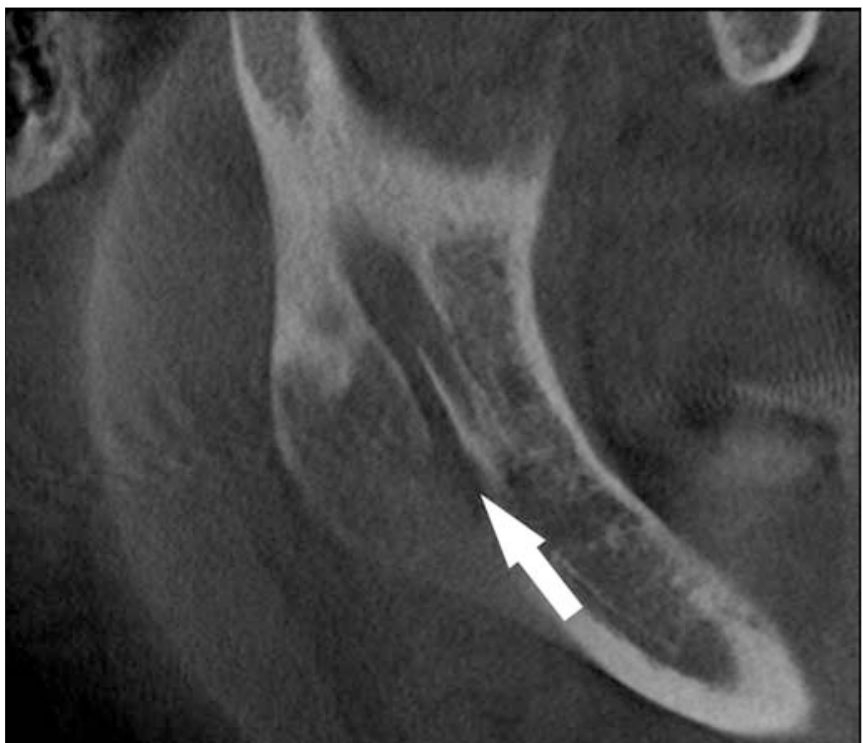

Fig. 5. Canal dentario inferior bífido tipo anterior sin convergencia.

CM bífidos presentados en este artículo, representan posiblemente canales sin fusionar que persistieron separados. Los CM bífidos tienen implicaciones clínicas importantes en cirugía de terceros molares, exodoncias de dientes posteriores en el maxilar inferior, tratamientos de conductos radiculares, cirugía sagital de rama (cirugía ortognática), obtención de injertos óseos en la zona retromolar y eventualmente 

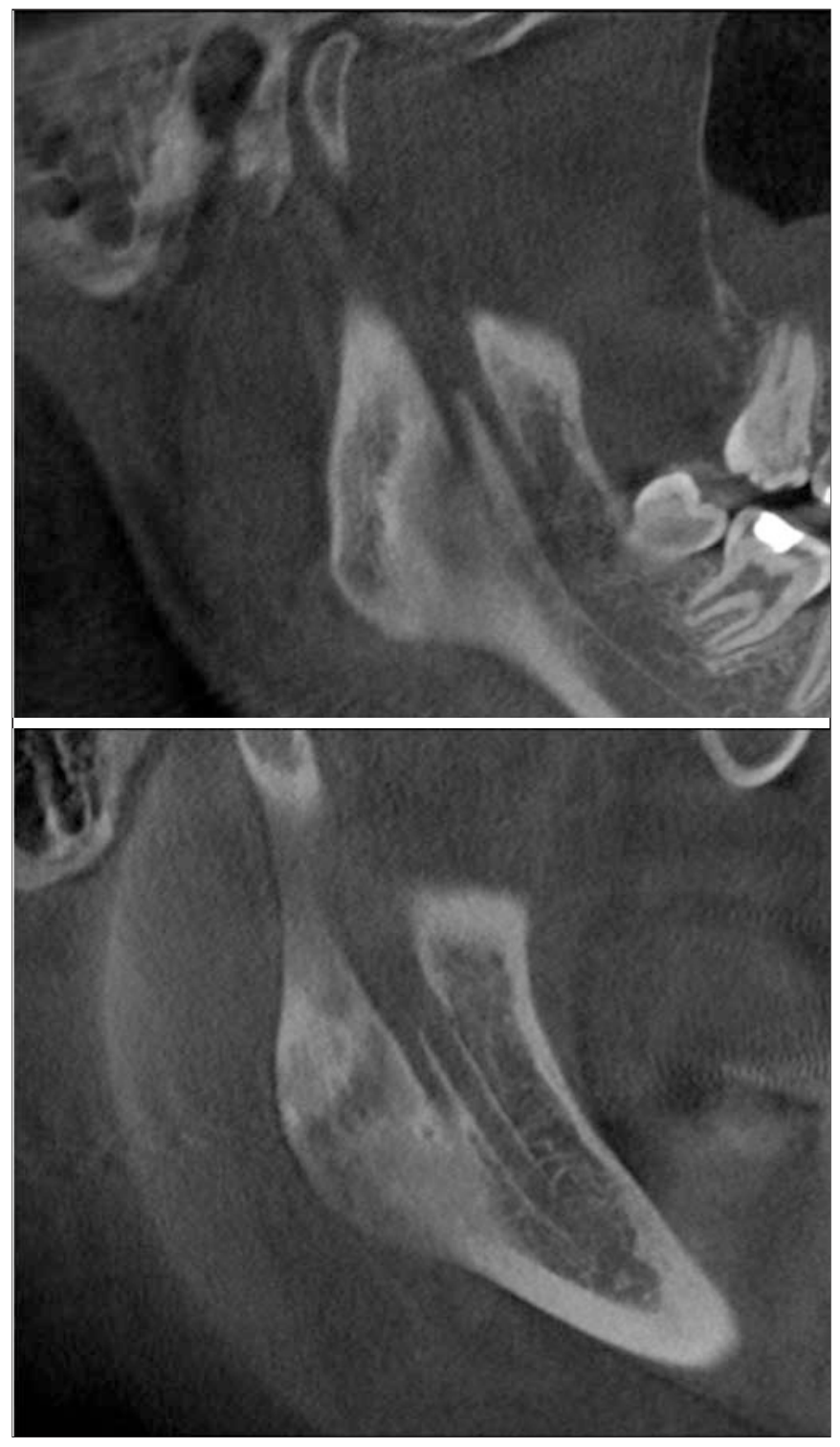

Figs. 6 y 7. Canales dentarios inferiores bífidos tipo anterior sin convergencia derechos.

como sitio de elección para la colocación de implantes oseointegrados. Adicionalmente, cuando existe algún tipo de bifurcación del CM se puede presentar anestesia inadecuada de la zona a intervenir, especialmente en aquellos casos con forámenes dentarios bífidos bilaterales.

La clasificación de los canales bífidos, particularmente el retromolar puede tener implicaciones clínicas importantes (4). Algunos autores han encontrado dentro del canal retromolar arterias y nervios deriva-

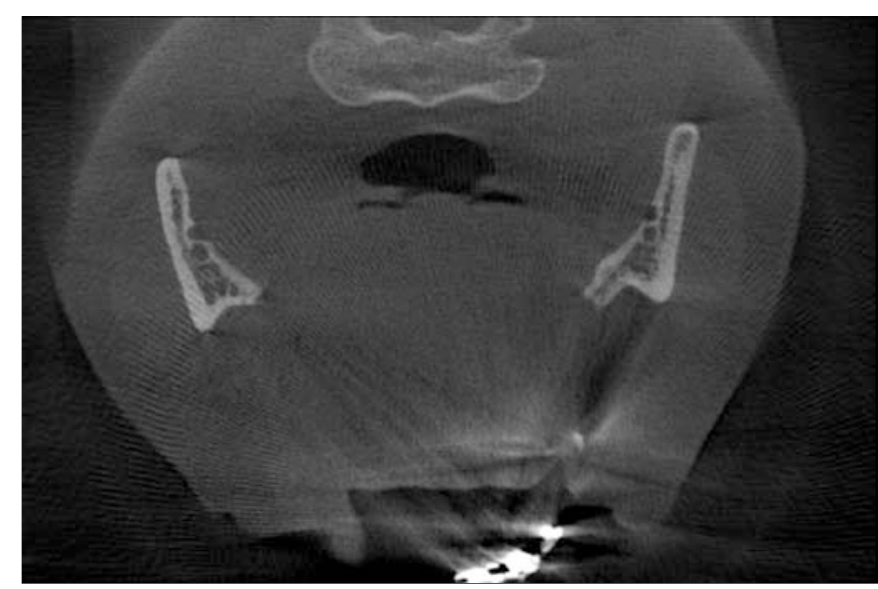

Fig. 8. Presencia de forámenes dentarios bífidos bilaterales.

dos de la arteria alveolar inferior y del nervio dentario inferior (6). Asimismo, la región retromolar se puede utilizar también como zona donante de injertos en bloque (7).

En esta serie de casos se observó mayor frecuencia de CM bífidos de tipo retromolar. En un estudio previo que utilizó TCHC este tipo de variante se observó en el $25.4 \%$ de los pacientes (4).

La radiografía panorámica presenta grandes limitaciones en el momento de evaluar variaciones anatómicas de los canales mandibulares debido a que es una imagen bidimensional. Es así como López-Videla y colaboradores (8) demostraron que en 84 pacientes estudiados, la radiografía panorámica solo identificó 7 casos con CM bífidos mientras que con la TCHC se observaron 55 ( $\mathrm{p}<0,001)$.

La identificación adecuada de CM múltiples le permitirá al profesional planear y llevar a cabo procedimientos clínicos adecuados, evitando correr riesgos mayores que comprometan la salud integral del paciente.

\section{BIBLIOGRAFÍA}

1. Rouas P, Nancy J, Bar D. Identification of double mandibular canals: Literature review and three case reports with CT scans and cone beam CT.Dentomaxillofac Radiol 2007;36:34-8. 
2. Grover PS, Lorton L. Bifid mandibular nerve as a possible cause of inadequate anesthesia in the mandible. J Oral Maxillofac Surg 1983;41: 177-9.

3. Langlais RP, Broadus R, Glass BJ. Bifid mandibular canals in panoramic radiographs. J Am Dent Assoc 1985;110:923-6.

4. Naitoh M, Hiraiwa Y, Aimiya H, Ariji E. Observation of bifid mandibular canal using cone-beam computerized tomography. Int J Oral Maxillofac Implants 2009;24:155-9.

5. Chavez-Lomeli ME,Mansilla Lory J, Pompa JA, Kjaer I. The human mandibular canal arises from three separate canals innervating different tooth groups. J Dent Res 1996;75:1540-4.

6. Kodera H, Hashimoto I. A case of mandibular retromolar canal: Elements of nerves and arteries in this canal. Kaibougaku Zashi 1995;70: 23-30.

7. Nkenke E, Radespiel-Troger M, Witfang J, SchultzeMosgau S, Winkler G, Neukam FW. Morbidity of harvesting of retromolar bone grafts: A prospective study. Clin Oral Impl Res 2002; 13:514-21.

8. López-Videla J, Vergara M, Rudolph M, Guzmán CL. Prevalencia de variables anatómicas en el recorrido de los conductos mandibulares. Estudio mediante tecnología Cone Beam. Rev Fac Odontol Univ Antioq 2010; 22: 23-32.

\section{CORRESPONDENCIA}

Carlos M. Ardila M

Calle 64, No 52-59

Medellín, Colombia.

e-mail: martinardila@gmail.com 